\title{
On Hadamard-type inequalities for $m$-convex functions via Riemann-Liouville fractional integrals
}

Ghulam Farid, Atiq Ur Rehman and Bushra Tariq

\begin{abstract}
In this paper we prove the Hadamard-type inequalities for $m$-convex functions via Riemann-Liouville fractional integrals and the Hadamard-type inequalities for convex functions via Riemann-Liouville fractional integral are deduced. Also we find connections with some well known results related to the Hadamard inequality.
\end{abstract}

Mathematics Subject Classification (2010): 26A51, 26A33, 26D10.

Keywords: Convex functions, Hadamard inequalities, fractional integrals.

\section{Introduction}

Following L'Hospital's and Leibniz's first inquiries, fractional calculus was primarily a study reserved for the best minds in mathematics. Fourier, Euler, Laplace were among those who were interested in fractional calculus and its mathematical consequences [15]. Euler and Liouville developed their thoughts about the computation of non-integer order integrals and derivatives. Many initiate, using their own notation and methodology, definitions that fit the concept of a non-integer order integral or derivative. The most well-known of these definitions that have been popularized in the subject of fractional calculus are the Riemann-Liouville and the Grunwald-Letnikov definition [4, 12]. In [18] Riemann-Liouville fractional integrals are defined as follows:

Definition 1.1. Let $f \in L_{1}[a, b]$. Then Riemann-Liouville fractional integrals of order $\alpha>0$ with $a \geq 0$ are defined as:

$$
J_{a+}^{\alpha} f(x)=\frac{1}{\Gamma(\alpha)} \int_{a}^{x}(x-t)^{\alpha-1} f(t) d t, \quad x>a
$$

and

$$
J_{b-}^{\alpha} f(x)=\frac{1}{\Gamma(\alpha)} \int_{x}^{b}(t-x)^{\alpha-1} f(t) d t, \quad x<b .
$$


For further details one may see $[15,16,17,9,8,13,19]$.

Convex functions play a vital role in the mathematical analysis. They have been considered for defining and finding new dimensions of analysis. In [20] Toader define the concept of $m$-convexity, an intermediate between usual convexity and star shape function.

Definition 1.2. A function $f:[0, b] \rightarrow \mathbb{R}, b>0$, is said to be $m$-convex, where $m \in[0,1]$, if we have

$$
f(t x+m(1-t) y) \leq t f(x)+m(1-t) f(y)
$$

for all $x, y \in[0, b]$ and $t \in[0,1]$.

If we take $m=1$, then we recapture the concept of convex functions defined on $[0, b]$ and if we take $m=0$, then we get the concept of starshaped functions on $[0, b]$. We recall that $f:[0, b] \rightarrow \mathbb{R}$ is called starshaped if

$$
f(t x) \leq t f(x) \text { for all } t \in[0,1] \text { and } x \in[0, b] .
$$

Denote by $K_{m}(b)$ the set of the $m$-convex functions on $[0, b]$ for which $f(0)<0$, then one has

$$
K_{1}(b) \subset K_{m}(b) \subset K_{0}(b),
$$

whenever $m \in(0,1)$. Note that in the class $K_{1}(b)$ are only convex functions $f:[0, b] \rightarrow$ $\mathbb{R}$ for which $f(0) \leq 0$ (see $[5])$.

Example 1.3. [14] The function $f:[0, \infty) \rightarrow \mathbb{R}$, given by

$$
f(x)=\frac{1}{12}\left(4 x^{3}-15 x^{2}+18 x-5\right)
$$

is $\frac{16}{17}$-convex function but it is not convex function.

For more results and inequalities related to $m$-convex functions one can consult for example $[7,5,11,2,16]$ along with references.

Let $f: I \rightarrow \mathbb{R}$ be a convex function on the interval $I$ of real numbers and $a, b \in I$ with $a<b$, then the following double inequality:

$$
f\left(\frac{a+b}{2}\right) \leq \frac{1}{b-a} \int_{a}^{b} f(x) d x \leq \frac{f(a)+f(b)}{2}
$$

is well known in literature as the Hadamard inequality.

For more refinements, generalizations and inequalities related to (1.3), see [1, 2, $3,16,6]$.

In [19], Sarikaya et al. proved the following Hadamard-type inequalities for Riemann-Liouville fractional integrals.

Theorem 1.4. Let $f:[a, b] \rightarrow \mathbb{R}$ be a positive function with $0 \leq a<b$ and $f \in L_{1}[a, b]$. If $f$ is a convex function on $[a, b]$, then the following inequalities for fractional integrals hold:

$$
f\left(\frac{a+b}{2}\right) \leq \frac{2^{\alpha-1} \Gamma(\alpha+1)}{(b-a)^{\alpha}}\left[J_{\left(\frac{a+b}{2}\right)+}^{\alpha} f(b)+J_{\left(\frac{a+b}{2}\right)-}^{\alpha} f(a)\right] \leq \frac{f(a)+f(b)}{2}
$$

with $\alpha>0$. 
Theorem 1.5. Let $f:[a, b] \rightarrow \mathbb{R}$ be a differentiable function on (a,b) with $a<b$. If $\left|f^{\prime}\right|^{q}$ is convex on $[a, b]$ for $q \geq 1$, then the following inequality for fractional integrals holds:

$$
\begin{aligned}
& \left|\frac{2^{\alpha-1} \Gamma(\alpha+1)}{(b-a)^{\alpha}}\left[J_{\left(\frac{a+b}{2}\right)+}^{\alpha} f(b)+J_{\left(\frac{a+b}{2}\right)-}^{\alpha} f(a)\right]-f\left(\frac{a+b}{2}\right)\right| \\
& \leq \frac{b-a}{4(\alpha+1)}\left(\frac{1}{2(\alpha+2)}\right)^{\frac{1}{q}}\left[\left((\alpha+1)\left|f^{\prime}(a)\right|^{q}+(\alpha+3)\left|f^{\prime}(b)\right|^{q}\right)^{\frac{1}{q}}\right. \\
& \left.+\left((\alpha+3)\left|f^{\prime}(a)\right|^{q}+(\alpha+1)\left|f^{\prime}(b)\right|^{q}\right)^{\frac{1}{q}}\right] .
\end{aligned}
$$

Theorem 1.6. Let $f:[a, b] \rightarrow \mathbb{R}$ be a differentiable function on (a,b) with $a<b$. If $\left|f^{\prime}\right|^{q}$ is convex on $[a, b]$ for $q>1$, then the following inequality for fractional integral holds:

$$
\begin{aligned}
& \left|\frac{2^{\alpha-1} \Gamma(\alpha+1)}{(b-a)^{\alpha}}\left[J_{\left(\frac{a+b}{2}\right)+}^{\alpha} f(b)+J_{\left(\frac{a+b}{2}\right)-}^{\alpha} f(a)\right]-f\left(\frac{a+b}{2}\right)\right| \\
& \leq \frac{b-a}{4}\left(\frac{1}{\alpha p+1}\right)^{\frac{1}{p}}\left[\left(\frac{\left|f^{\prime}(a)\right|^{q}+3\left|f^{\prime}(b)\right| q}{4}\right)^{\frac{1}{q}}+\left(\frac{3\left|f^{\prime}(a)\right|^{q}+\left|f^{\prime}(b)\right|^{q}}{4}\right)^{\frac{1}{q}}\right] \\
& \leq \frac{b-a}{4}\left(\frac{4}{\alpha p+1}\right)^{\frac{1}{p}}\left[\left|f^{\prime}(a)\right|+\left|f^{\prime}(b)\right|\right],
\end{aligned}
$$

where $\frac{1}{p}+\frac{1}{q}=1$.

In this paper we generalize the fractional Hadamard-type inequalities (1.4), (1.5) and (1.6) for $m$-convex function via Riemann-Liouville fractional integrals and show that these inequalities are the special cases of our results. Also we find some well known results.

\section{Hadamard-type inequalities for $m$-convex functions via fractional integrals}

Start with the following result.

Theorem 2.1. Let $f:[a, b] \rightarrow \mathbb{R}$ be a positive function with $0 \leq a<b$ and $f \in L_{1}[a, b]$. If $f$ is a m-convex function on $[a, b]$, then the following inequalities for fractional integrals hold:

$$
\begin{aligned}
& f\left(\frac{a+m b}{2}\right) \leq \frac{2^{\alpha-1} \Gamma(\alpha+1)}{(m b-a)^{\alpha}}\left[J_{\left(\frac{a+m b}{2}\right)+}^{\alpha} f(m b)+m^{\alpha+1} J_{\left(\frac{a+m b}{2 m}\right)-}^{\alpha} f\left(\frac{a}{m}\right)\right] \\
& \leq \frac{\alpha}{4(\alpha+1)}\left[f(a)-m^{2} f\left(\frac{a}{m^{2}}\right)\right]+\frac{m}{2}\left[f(b)+m f\left(\frac{a}{m^{2}}\right)\right]
\end{aligned}
$$

with $\alpha>0$.

Proof. From $m$-convexity of $f$ we have,

$$
f\left(\frac{x+m y}{2}\right) \leq \frac{f(x)+m f(y)}{2} .
$$


Put $x=\frac{t}{2} a+m \frac{(2-t)}{2} b, y=\frac{(2-t)}{2 m} a+\frac{t}{2} b$ for $t \in[0,1]$. Then $x, y \in[a, b]$ and above inequality gives,

$$
2 f\left(\frac{a+m b}{2}\right) \leq f\left(\frac{t}{2} a+m \frac{2-t}{2} b\right)+m f\left(\frac{2-t}{2 m} a+\frac{t}{2} b\right),
$$

multiplying both sides of above inequality with $t^{\alpha-1}$, and integrating over $[0,1]$ we have,

$$
\begin{aligned}
& \frac{2}{\alpha} f\left(\frac{a+m b}{2}\right) \\
& \leq \int_{0}^{1} t^{\alpha-1} f\left(\frac{t}{2} a+m \frac{2-t}{2} b\right) d t+m \int_{0}^{1} t^{\alpha-1} f\left(\frac{2-t}{2 m} a+\frac{t}{2} b\right) d t \\
& =\int_{m b}^{\frac{a+m b}{2}}\left(\frac{2}{m b-a}(m b-u)\right)^{\alpha-1} f(u) \frac{2 d u}{a-m b} \\
& +m^{2} \int_{\frac{a}{m}}^{\frac{a+m b}{2 m}}\left(\frac{2}{b-\frac{a}{m}}\left(v-\frac{a}{m}\right)\right)^{\alpha-1} f(v) \frac{2 d v}{m b-a} \\
& =\frac{2^{\alpha} \Gamma(\alpha)}{(m b-a)^{\alpha}}\left[J_{\left(\frac{a+m b}{2}\right)+}^{\alpha} f(m b)+m^{\alpha+1} J_{\left(\frac{a+m b}{2 m}\right)-}^{\alpha} f\left(\frac{a}{m}\right)\right]
\end{aligned}
$$

from which one has

$$
f\left(\frac{a+m b}{2}\right) \leq \frac{2^{\alpha-1} \Gamma(\alpha+1)}{(m b-a)^{\alpha}}\left[J_{\left(\frac{a+m b}{2}\right)+}^{\alpha} f(m b)+m^{\alpha+1} J_{\left(\frac{a+m b}{2 m}\right)-}^{\alpha} f\left(\frac{a}{m}\right)\right] .
$$

On the other hand $m$-convexity of $f$ gives

$$
\begin{aligned}
& f\left(\frac{t}{2} a+m \frac{2-t}{2} b\right)+m f\left(\frac{2-t}{2 m} a+\frac{t}{2} b\right) \\
& \leq \frac{t}{2}\left[f(a)-m^{2} f\left(\frac{a}{m^{2}}\right)\right]+m\left[f(b)+m f\left(\frac{a}{m^{2}}\right)\right],
\end{aligned}
$$

multiplying both sides of above inequality with $t^{\alpha-1}$, and integrating over $[0,1]$ we have,

$$
\begin{aligned}
& \int_{0}^{1} t^{\alpha-1} f\left(\frac{t}{2} a+m \frac{2-t}{2} b\right) d t+m \int_{0}^{1} t^{\alpha-1} f\left(\frac{2-t}{2 m} a+\frac{t}{2} b\right) d t \\
& \leq \frac{1}{2}\left[f(a)-m^{2} f\left(\frac{a}{m^{2}}\right)\right] \int_{0}^{1} t^{\alpha} d t+m\left[f(b)+m f\left(\frac{a}{m^{2}}\right)\right] \int_{0}^{1} t^{\alpha-1} d t \\
& \int_{m b}^{\frac{a+m b}{2}}\left(\frac{2}{m b-a}(m b-u)\right)^{\alpha-1} f(u) \frac{2 d u}{a-m b} \\
& +m^{2} \int_{\frac{a}{m}}^{\frac{a+m b}{2 m}}\left(\frac{2}{b-\frac{a}{m}}\left(v-\frac{a}{m}\right)\right)^{\alpha-1} f(v) \frac{2 d v}{m b-a} \\
& \leq \frac{1}{2(\alpha+1)}\left[f(a)-m^{2} f\left(\frac{a}{m^{2}}\right)\right]+\frac{m}{\alpha}\left[f(b)+m f\left(\frac{a}{m^{2}}\right)\right]
\end{aligned}
$$


from which one has

$$
\begin{aligned}
& \frac{2^{\alpha-1} \Gamma(\alpha+1)}{(m b-a)^{\alpha}}\left[J_{\left(\frac{a+m b}{2}\right)+}^{\alpha} f(m b)+m^{\alpha+1} J_{\left(\frac{a+m b}{2 m}\right)-}^{\alpha} f\left(\frac{a}{m}\right)\right] \\
& \leq \frac{\alpha}{4(\alpha+1)}\left[f(a)-m^{2} f\left(\frac{a}{m^{2}}\right)\right]+\frac{m}{2}\left[f(b)+m f\left(\frac{a}{m^{2}}\right)\right] .
\end{aligned}
$$

Combining inequality (2.4) and inequality (2.5) we get inequality (2.1) .

Remark 2.2. If we take $m=1$, Theorem 2.1 gives inequality (1.4) of Theorem 1.4 and putting $\alpha=1$ along with $m=1$ in Theorem 2.1 we get the classical Hadamard inequality.

For next results we need the following lemma.

Lemma 2.3. Let $f:[a, b] \rightarrow \mathbb{R}$ be a differentiable mapping on $(a, b)$ with $a<b$. If $f^{\prime} \in L[a, b]$, then the following equality for fractional integrals holds:

$$
\begin{aligned}
& \frac{2^{\alpha-1} \Gamma(\alpha+1)}{(m b-a)^{\alpha}}\left[J_{\left(\frac{a+m b}{2}\right)+}^{\alpha} f(m b)+m^{\alpha+1} J_{\left(\frac{a+m b}{2 m}\right)-}^{\alpha} f\left(\frac{a}{m}\right)\right] \\
& -\frac{1}{2}\left[f\left(\frac{a+m b}{2}\right)+m f\left(\frac{a+m b}{2 m}\right)\right] \\
& =\frac{m b-a}{4}\left[\int_{0}^{1} t^{\alpha} f^{\prime}\left(\frac{t}{2} a+m \frac{2-t}{2} b\right) d t-\int_{0}^{1} t^{\alpha} f^{\prime}\left(\frac{2-t}{2 m} a+\frac{t}{2} b\right) d t\right] .
\end{aligned}
$$

Proof. One can note that

$$
\begin{aligned}
& \frac{m b-a}{4}\left[\int_{0}^{1} t^{\alpha} f^{\prime}\left(\frac{t}{2} a+m \frac{2-t}{2} b\right) d t\right] \\
& =\frac{m b-a}{4}\left[-\frac{2}{m b-a} f\left(\frac{a+m b}{2}\right)\right. \\
& \left.-\frac{2 \alpha}{(a-m b)} \int_{m b}^{\frac{a+m b}{2}}\left(\frac{2}{m b-a}(m b-x)\right)^{\alpha-1} \frac{2}{a-m b} f(x) d x\right] \\
& =\frac{m b-a}{4}\left[-\frac{2}{m b-a} f\left(\frac{a+m b}{2}\right)+\frac{2^{\alpha+1} \Gamma(\alpha+1)}{(m b-a)^{\alpha+1}} J_{\left(\frac{a+m b}{2}\right)-}^{\alpha} f(m b)\right] .
\end{aligned}
$$

Similarly

$$
\begin{aligned}
& -\frac{m b-a}{4}\left[\int_{0}^{1} t^{\alpha} f^{\prime}\left(\frac{2-t}{2 m} a+\frac{t}{2} b\right) d t\right] \\
& =-\frac{m b-a}{4}\left[\frac{2 m}{m b-a} f\left(\frac{a+m b}{2 m}\right)-\frac{2^{\alpha+1} m^{\alpha+1} \Gamma(\alpha+1)}{(m b-a)^{\alpha+1}} J_{\left(\frac{a+m b}{2 m}\right)+}^{\alpha} f\left(\frac{a}{m}\right)\right] .
\end{aligned}
$$

Adding (2.7) and (2.9) one has (2.6).

Using the above lemma we give the following Hadamard-type inequality. 
Theorem 2.4. Let $f:[a, b] \rightarrow \mathbb{R}$ be a differentiable function on (a,b) with $a<b$. If $\left|f^{\prime}\right|^{q}$ is $m$-convex on $[a, b]$ for $q \geq 1$, then the following inequality for fractional integrals holds:

$$
\begin{aligned}
& \mid \frac{2^{\alpha-1} \Gamma(\alpha+1)}{(m b-a)^{\alpha}}\left[J_{\left(\frac{a+m b}{2}\right)+}^{\alpha} f(m b)+m^{\alpha+1} J_{\left(\frac{a+m b}{2 m}\right)-}^{\alpha} f\left(\frac{a}{m}\right)\right] \\
& -\frac{1}{2}\left[f\left(\frac{a+m b}{2}\right)+m f\left(\frac{a+m b}{2 m}\right)\right] \mid \\
& \leq \frac{m b-a}{4(\alpha+1)}\left(\frac{1}{2(\alpha+2)}\right)^{\frac{1}{q}}\left[\left((\alpha+1)\left|f^{\prime}(a)\right|^{q}+m(\alpha+3)\left|f^{\prime}(b)\right|^{q}\right)^{\frac{1}{q}}\right. \\
& \left.+\left(m(\alpha+3)\left|f^{\prime}\left(\frac{a}{m^{2}}\right)\right|^{q}+(\alpha+1)\left|f^{\prime}(b)\right|^{q}\right)^{\frac{1}{q}}\right] .
\end{aligned}
$$

with $\alpha>0$.

Proof. From Lemma 2.3 and $m$-convexity of $\left|f^{\prime}\right|^{q}$ and for $q=1$ we have

$$
\begin{aligned}
& \mid \frac{2^{\alpha-1} \Gamma(\alpha+1)}{(m b-a)^{\alpha}}\left[J_{\left(\frac{a+m b}{2}\right)+}^{\alpha} f(m b)+m^{\alpha+1} J_{\left(\frac{a+m b}{2 m}\right)-}^{\alpha} f\left(\frac{a}{m}\right)\right] \\
& -\frac{1}{2}\left[f\left(\frac{a+m b}{2}\right)+m f\left(\frac{a+m b}{2 m}\right)\right] \mid \\
& \leq \frac{m b-a}{4} \int_{0}^{1} t^{\alpha}\left(\left|f^{\prime}\left(\frac{t}{2} a+m \frac{2-t}{2} b\right)\right| d t+\left|f^{\prime}\left(\frac{2-t}{2 m} a+\frac{t}{2} b\right)\right|\right) d t . \\
& =\frac{m b-a}{4}\left(\frac{m}{\alpha+1}\left[\left|f^{\prime}(b)\right|+\left|f^{\prime}\left(\frac{a}{m^{2}}\right)\right|\right]\right. \\
& \left.+\left[\left|f^{\prime}(a)\right|-m\left|f^{\prime}\left(\frac{a}{m^{2}}\right)\right|+\left|f^{\prime}(b)\right|-m\left|f^{\prime}(b)\right|\right]\right) .
\end{aligned}
$$

For $q>1$ we proceed as follows. Using Lemma 2.3 we have

$$
\begin{aligned}
& \mid \frac{2^{\alpha-1} \Gamma(\alpha+1)}{(m b-a)^{\alpha}}\left[J_{\left(\frac{a+m b}{2}\right)+}^{\alpha} f(m b)+m^{\alpha+1} J_{\left(\frac{a+m b}{2 m}\right)-}^{\alpha} f\left(\frac{a}{m}\right)\right] \\
& -\frac{1}{2}\left[f\left(\frac{a+m b}{2}\right)+m f\left(\frac{a+m b}{2 m}\right)\right] \mid \\
& \leq \frac{m b-a}{4} \int_{0}^{1} t^{\alpha}\left|f^{\prime}\left(\frac{t}{2} a+m \frac{2-t}{2} b\right)\right| d t+\int_{0}^{1} t^{\alpha}\left|f^{\prime}\left(\frac{2-t}{2 m} a+\frac{t}{2} b\right)\right| d t .
\end{aligned}
$$

Using power mean inequality we get

$$
\begin{aligned}
& \mid \frac{2^{\alpha-1} \Gamma(\alpha+1)}{(m b-a)^{\alpha}}\left[J_{\left(\frac{a+m b}{2}\right)+}^{\alpha} f(m b)+m^{\alpha} J_{\left(\frac{a+m b}{2 m}\right)-}^{\alpha} f\left(\frac{a}{m}\right)\right] \\
& -\frac{1}{2}\left[f\left(\frac{a+m b}{2}\right)+m f\left(\frac{a+m b}{2 m}\right)\right] \mid \\
& \leq \frac{m b-a}{4}\left(\frac{1}{\alpha+1}\right)^{\frac{1}{p}}\left[\left[\int_{0}^{1} t^{\alpha}\left|f^{\prime}\left(\frac{t}{2} a+m \frac{2-t}{2} b\right)\right|^{q} d t\right]^{\frac{1}{q}}\right.
\end{aligned}
$$




$$
\left.+\left[\int_{0}^{1} t^{\alpha}\left|f^{\prime}\left(\frac{2-t}{2 m} a+\frac{t}{2} b\right)\right|^{q} d t\right]^{\frac{1}{q}}\right] .
$$

$m$-convexity of $\left|f^{\prime}\right|^{q}$ gives

$$
\begin{aligned}
& \mid \frac{2^{\alpha-1} \Gamma(\alpha+1)}{(m b-a)^{\alpha}}\left[J_{\left(\frac{a+m b}{2}\right)+}^{\alpha} f(m b)+m^{\alpha+1} J_{\left(\frac{a+m b}{2 m}\right)-}^{\alpha} f\left(\frac{a}{m}\right)\right] \\
& -\frac{1}{2}\left[f\left(\frac{a+m b}{2}\right)+m f\left(\frac{a+m b}{2 m}\right)\right] \mid \\
& \leq \frac{m b-a}{4}\left(\frac{1}{\alpha+1}\right)^{\frac{1}{p}}\left[\left[\int_{0}^{1} t^{\alpha}\left(\frac{t}{2}\left|f^{\prime}(a)\right|^{q}+m \frac{2-t}{2}\left|f^{\prime}(b)\right|^{q}\right) d t\right]^{\frac{1}{q}}\right. \\
& \left.+\left[\int_{0}^{1} t^{\alpha}\left(m \frac{2-t}{2}\left|f^{\prime}\left(\frac{a}{m^{2}}\right)\right|^{q}+\frac{t}{2}\left|f^{\prime}(b)\right|^{q}\right) d t\right]^{\frac{1}{q}}\right] \\
& =\frac{m b-a}{4(\alpha+1)}\left(\frac{1}{2(\alpha+2)}\right)^{\frac{1}{q}}\left[\left((\alpha+1)\left|f^{\prime}(a)\right|^{q}+m(\alpha+3)\left|f^{\prime}(b)\right|^{q}\right)^{\frac{1}{q}}\right. \\
& \left.+\left(m(\alpha+3)\left|f^{\prime}\left(\frac{a}{m^{2}}\right)\right|^{q}+(\alpha+1)\left|f^{\prime}(b)\right|^{q}\right)^{\frac{1}{q}}\right] .
\end{aligned}
$$

Hence the proof is complete.

Remark 2.5. If we take $m=1$ in Theorem 2.4, we get inequality (1.5) of Theorem 1.5 and if we take $\alpha=q=1$ along with $m=1$ in Theorem 2.4 , then inequality $(2.10)$ gives the following result.

Corollary 2.6. With the assumptions of Theorem 2.4 we have

$$
\left|\frac{1}{b-a} \int_{a}^{b} f(x) d x-f\left(\frac{a+b}{2}\right)\right| \leq \frac{(b-a)}{8}\left(\left|f^{\prime}(a)\right|+\left|f^{\prime}(b)\right|\right) .
$$

Theorem 2.7. Let $f:[a, b] \rightarrow \mathbb{R}$ be a differentiable function on (a,b) with $a<b$. If $\left|f^{\prime}\right|^{q}$ is $m$-convex on $[a, b]$ for $q>1$, then the following inequality for fractional integral holds:

$$
\begin{aligned}
& \mid \frac{2^{\alpha-1} \Gamma(\alpha+1)}{(m b-a)^{\alpha}}\left[J_{\left(\frac{a+m b}{2}\right)+}^{\alpha} f(m b)+m^{\alpha+1} J_{\left(\frac{a+m b}{2 m}\right)-}^{\alpha} f\left(\frac{a}{m}\right)\right] \\
& -\frac{1}{2}\left[f\left(\frac{a+m b}{2}\right)+m f\left(\frac{a+m b}{2 m}\right)\right] \mid \\
& \leq \frac{m b-a}{4}\left(\frac{1}{\alpha p+1}\right)^{\frac{1}{p}}\left[\left(\frac{\left|f^{\prime}(a)\right|^{q}+3 m\left|f^{\prime}(b)\right|^{q}}{4}\right)^{\frac{1}{q}}+\left(\frac{3 m\left|f^{\prime}\left(\frac{a}{m^{2}}\right)\right|^{q}+\left|f^{\prime}(b)\right|^{q}}{4}\right)^{\frac{1}{q}}\right] \\
& \leq \frac{m b-a}{4}\left(\frac{4}{\alpha p+1}\right)^{\frac{1}{p}}\left[\left|f^{\prime}(a)\right|+\left|f^{\prime}(b)\right|+3 m\left(\left|f^{\prime}\left(\frac{a}{m^{2}}\right)\right|+\left|f^{\prime}(b)\right|\right)\right]
\end{aligned}
$$

with $\frac{1}{p}+\frac{1}{q}=1$. 
Proof. Using Lemma 2.3 we have

$$
\begin{aligned}
& \mid \frac{2^{\alpha-1} \Gamma(\alpha+1)}{(m b-a)^{\alpha}}\left[J_{\left(\frac{a+m b}{2}\right)+}^{\alpha} f(m b)+m^{\alpha+1} J_{\left(\frac{a+m b}{2 m}\right)-}^{\alpha} f\left(\frac{a}{m}\right)\right] \\
& -\frac{1}{2}\left[f\left(\frac{a+m b}{2}\right)+m f\left(\frac{a+m b}{2 m}\right)\right] \mid \\
& \leq \frac{m b-a}{4}\left[\int_{0}^{1} t^{\alpha}\left|f^{\prime}\left(\frac{t}{2} a+m \frac{2-t}{2} b\right)\right| d t+\int_{0}^{1} t^{\alpha}\left|f^{\prime}\left(\frac{2-t}{2 m} a+\frac{t}{2} b\right)\right| d t\right] .
\end{aligned}
$$

From the Hölder's inequality we get

$$
\begin{aligned}
& \mid \frac{2^{\alpha-1} \Gamma(\alpha+1)}{(m b-a)^{\alpha}}\left[J_{\left(\frac{a+m b}{2}\right)+}^{\alpha} f(m b)+m^{\alpha+1} J_{\left(\frac{a+m b}{2 m}\right)-}^{\alpha} f\left(\frac{a}{m}\right)\right] \\
& -\frac{1}{2}\left[f\left(\frac{a+m b}{2}\right)+m f\left(\frac{a+m b}{2 m}\right)\right] \mid \\
& \leq \frac{m b-a}{4}\left[\left[\int_{0}^{1} t^{\alpha p} d t\right]^{\frac{1}{p}}\left[\int_{0}^{1}\left|f^{\prime}\left(\frac{t}{2} a+m \frac{2-t}{2} b\right)\right|^{q} d t\right]^{\frac{1}{q}}\right. \\
& \left.+\left[\int_{0}^{1} t^{\alpha p} d t\right]^{\frac{1}{p}}\left[\int_{0}^{1}\left|f^{\prime}\left(\frac{2-t}{2 m} a+\frac{t}{2} b\right)\right|^{q} d t\right]^{\frac{1}{q}}\right] .
\end{aligned}
$$

$m$-convexity of $\left|f^{\prime}\right|^{q}$ gives

$$
\begin{aligned}
& \mid \frac{2^{\alpha-1} \Gamma(\alpha+1)}{(m b-a)^{\alpha}}\left[J_{\left(\frac{a+m b}{2}\right)+}^{\alpha} f(m b)+m^{\alpha+1} J_{\left(\frac{a+m b}{2 m}\right)-}^{\alpha} f\left(\frac{a}{m}\right)\right] \\
& -\frac{1}{2}\left[f\left(\frac{a+m b}{2}\right)+m f\left(\frac{a+m b}{2 m}\right)\right] \mid \\
& \leq \frac{m b-a}{4}\left(\frac{1}{\alpha p+1}\right)^{\frac{1}{p}}\left[\left[\int_{0}^{1}\left(\frac{t}{2}\left|f^{\prime}(a)\right|^{q}+m \frac{2-t}{2}\left|f^{\prime}(b)\right|^{q}\right) d t\right]^{\frac{1}{q}}\right. \\
& \left.+\left[\int_{0}^{1}\left(m \frac{2-t}{2}\left|f^{\prime}\left(\frac{a}{m^{2}}\right)\right|^{q}+\frac{t}{2}\left|f^{\prime}(b)\right|^{q}\right) d t\right]^{\frac{1}{q}}\right] \\
& =\frac{m b-a}{4}\left(\frac{1}{\alpha p+1}\right)^{\frac{1}{p}}\left[\left[\frac{\left|f^{\prime}(a)\right|^{q}+3 m\left|f^{\prime}(b)\right|^{q}}{4}\right]^{\frac{1}{q}}+\left[\frac{3 m\left|f^{\prime}\left(\frac{a}{m^{2}}\right)\right|^{q}+\left|f^{\prime}(b)\right|^{q}}{4}\right]^{\frac{1}{q}}\right] .
\end{aligned}
$$

For the second inequality of (2.12) we use Minkowski's inequality as

$$
\begin{gathered}
\mid \frac{2^{\alpha-1} \Gamma(\alpha+1)}{(m b-a)^{\alpha}}\left[J_{\left(\frac{a+m b}{2}\right)+}^{\alpha} f(m b)+m^{\alpha+1} J_{\left(\frac{a+m b}{2 m}\right)-}^{\alpha} f\left(\frac{a}{m}\right)\right] \\
-\frac{1}{2}\left[f\left(\frac{a+m b}{2}\right)+m f\left(\frac{a+m b}{2 m}\right)\right] \mid \\
\leq \frac{m b-a}{16}\left(\frac{4}{\alpha p+1}\right)^{\frac{1}{p}}\left[\left[\left|f^{\prime}(a)\right|^{q}+3 m\left|f^{\prime}(b)\right|^{q}\right]^{\frac{1}{q}}+\left[3 m\left|f^{\prime}\left(\frac{a}{m^{2}}\right)\right|^{q}+\left|f^{\prime}(b)\right|^{q}\right]^{\frac{1}{q}}\right]
\end{gathered}
$$




$$
\leq \frac{m b-a}{4}\left(\frac{4}{\alpha p+1}\right)^{\frac{1}{p}}\left[\left|f^{\prime}(a)\right|+\left|f^{\prime}(b)\right|+3 m\left(\left|f^{\prime}\left(\frac{a}{m^{2}}\right)\right|+\left|f^{\prime}(b)\right|\right)\right] .
$$

Remark 2.8. If we take $m=1$ in Theorem 2.7, we get inequality (1.6) of Theorem 1.6 and if we take $\alpha=1$ along with $m=1$ in Theorem 2.7, then inequality (2.12) gives the following result.

Corollary 2.9. With the assumptions of Theorem 2.7 we have

$$
\begin{aligned}
& \left|\frac{1}{b-a} \int_{a}^{b} f(x) d x-f\left(\frac{a+b}{2}\right)\right| \\
& \leq \frac{b-a}{16}\left(\frac{4}{p+1}\right)^{\frac{1}{p}}\left[\left(\left|f^{\prime}(a)\right|^{q}+3\left|f^{\prime}(b)\right|^{q}\right)^{\frac{1}{q}}+\left(3\left|f^{\prime}(a)\right|^{q}+\left|f^{\prime}(b)\right|^{q}\right)^{\frac{1}{q}}\right] .
\end{aligned}
$$

\section{References}

[1] Azpeitia, A.G., Convex functions and the Hadamard inequality, Revista Colombiana Mat., 28(1994), 7-12.

[2] Bakula, M.K., Ozdemir, M.E., Pečarić, J., Hadamard type inequalities for $m$-convex and $(\alpha, m)$-convex functions, J. Ineq. Pure Appl. Math., 9(2008), no. 4, art. 96.

[3] Bakula, M.K., Pečarić, J., Note on some Hadamard-type inequalities, J. Ineq. Pure Appl. Math., 5(2004), no. 3, art. 74.

[4] David, S.A., Linares, J.L., Pallone, E.M.J.A., Fractional order calculus: historical apologia, basic concepts and some applications, Rev. Bras. Ensino Fs., 33(2011), no. 4, 43024302.

[5] Dragomir, S.S., On some new inequalities of Hermite-Hadamard type for $m$-convex functions, Tamkang J. Math., 33(2002), no. 1, 45-56.

[6] Farid, G., Rehman, A.U., Zahra, M., On Hadamard inequalities for relative convex functions via fractional integrals, Nonlinear Anal. Forum, 21(2016), no. 1, 77-86.

[7] Farid, G., Marwan, M., Rehman, A.U., New mean value theorems and generalization of Hadamard inequality via coordinated m-convex functions, J. Inequal. Appl. 2015, Article ID 283, 2015, 11p.

[8] Gorenflo, R., Mainardi, F., Fractional calculus: integral and differential equations of fractional order, Springer Verlag, Wien, 1997, 223-276.

[9] Hilfer, R., (ed.), Applications of fractional calculus in physics, World Scientific Publishing Co. 2000.

[10] Kirmaci, U.S., Bakula, M.K., Ozdemir, M.E., Pečarić, J., Hadamard-tpye inequalities for s-convex functions, Appl. Math. Comput., 193(2007), 26-35.

[11] Bakula, M.K., Pečarić, J., Ribicic, M., Companion inequalities to Jensen's inequality for $m$-convex and $(\alpha, m)$-convex functions, J. Inequal. Pure Appl. Math., 7(2006), art. 194, online:htpp://jipam.vu.edu.au.

[12] Loverro, A., Fractional calculus: history, definitions and applications for the engineer, Department of Aerospace and Mechanical Engineering, University of Notre Dame, 2004.

[13] Miller, S., Ross, B., An introduction to fractional calculus and fractional differential equations, John Wiley And Sons, USA, 1993. 
[14] Mocanu, P.T., Serb, I., Toader, G., Real star-convex functions, Studia Univ. BabeşBolyai Math., 42(1997), no. 3, 65-80.

[15] Nishimoto, K., An essence of Nishimoto's fractional calculus, Descartes Press Co., 1991.

[16] Özdemir, M.E., Avci, M., Set, E., On some inequalities of Hermite-Hadamard type via m-convexity, Appl. Math. Lett., 23(2010), no. 9, 1065-1070.

[17] Podlubny, I., Fractional differential equations, Mathematics in Science and Engineering V198, Academic Press 1999.

[18] Sarikaya, M.Z., Set, E., Yaldiz, H., Basak, N., Hermite-Hadamard's inequalities for fractional integrals and related fractional inequalities, J. Math. Comput. Model., 57(2013), 2403-2407.

[19] Sarikaya, M.Z., Yildirim, H., On Hermite-Hadamard type inequalities for RiemannLiouville fractional integrals, RGMIA Research Report Collection, 17(98)(2014), 10 pp.

[20] Toader, G.H., Some generalizations of convexity, Proc. Colloq. Approx. Optim., 1984, 329-338.

Ghulam Farid

COMSATS Institute of Information Technology

Attock Campus, Pakistan

e-mail: faridphdsms@hotmail.com

ghlmfarid@ciit-attock.edu.pk

Atiq Ur Rehman

COMSATS Institute of Information Technology

Attock, Pakistan

e-mail: atiq@mathcity.org

Bushra Tariq

Department of Mathematics

Comsats Institute of Information Technology

Attock, Pakistan

e-mail: bushratariq38@yahoo.com 